Pacific Journal of Mathematics

TOPOLOGIES ON THE SET OF CLOSED SUBSETS 


\title{
TOPOLOGIES ON THE SET OF CLOSED SUBSETS
}

\author{
FRANK WATTENBERG
}

In this paper the techniques of Nonstandard Analysis are used to study topologies on the set $\Gamma(X)$ of closed subsets of a topological space $X$. The first section of the paper investigates the "compact" topology developed by Narens and constructs a variant of that topology which is particularly useful for non locally compact spaces $X$. (When $X$ is locally compact this variant is shown to be identical with Naren's original "compact" topology.) This new topology is a natural extension to $\Gamma(X)$ of the one point compactification of $X$ embedded in $\Gamma(X)$ in the obvious way with the point at infinity corresponding to the empty set. The second section shows that the techniques developed by Narens can be used to obtain a natural characterization of the Vietoris Topology by considering monads of non nearstandard points. The final section uses this same approach to construct a topological analog of the Hausdorff metric for normal spaces.

0. Introduction. Suppose that $X$ is a topological space and that $\Gamma$ denotes the set of closed subsets of $X$. It is frequently desirable to endow $\Gamma$ with a topology of its own. Various topologies on $\Gamma$ have been proposed and studied by several mathematicians. If $X$ is a metric space, Hausdorff (see [2], [6], [7]) defined a metric on $\Gamma$ in a natural way. With this metric $X$ is embedded isometrically as a closed subset of $\Gamma$ by the mapping $x \mapsto\{x\}$. One drawback of this metric, however, is that it depends in an essential way on the metric on $X$. That is, $d$ and $d^{\prime}$ may be two metrics for the same topology on $X$, but induce Hausdorff metrics which do not give the same topology on $\Gamma$. In [10] E. Michaels investigates among other topologies the Vietoris or Finite topology on $\Gamma$. This topology also has the property that $X$ is embedded as a closed subset of $\Gamma$ by the mapping $x \mapsto\{x\}$. Both the Hausdorff metric on $\Gamma$ and the Vietoris topology on $\Gamma$ make $\Gamma$ into a compact space if and only if $X$ was originally compact. More recently, L. Narens [12] has introduced an interesting topology on $\Gamma$ using the techniques of Nonstandard Analysis. This topology always ma!.es $\Gamma$ a compact set with the empty set $\varnothing \in \Gamma$ acting (see Theorem I.8, somewhat like the point at infinity of the one-point compactification of $X$.

Nonstandard Analysis provides a particularly nice framework for investigating topological questions. Intuitively, a topological space is a set together with some notion of "nearness" 
If $X$ is any set and ${ }^{*} X$ is a nonstandard extension of $X$ then a topology on $X$ can be described by a relationship of "infinitely close" on some points of ${ }^{*} X$ (see [9], [13], [14]). If $X$ is a topological space, $x \in X$ and $y \in{ }^{*} X$ we say $y$ is infinitely close to $x$, written $y \sim x$ or $y \in \mu(x)$, provided for every standard open set $\mathcal{O}$ if $x \in \mathcal{O}$ then $y \in{ }^{*} \mathcal{O}$. In this case $x$ is called the standard part of $y$, denoted $x=\operatorname{St}(y)$. If $A$ is an internal subset of ${ }^{*} X$, let $\operatorname{St}(A)=\{x \in X \mid \mu(x) \cap A \neq \varnothing\}$. Under suitable conditions on ${ }^{*} X \operatorname{St}(A)$ is always closed. Now, if $A, B \in{ }^{*} X$, Narens defines $A \sim B$ provided $\operatorname{St}(A)=\operatorname{St}(B)$. He uses this relationship to define a topology which he calls the compact topology. In the present paper we will call this same topology the $N$-compact topology. Although the relationship $\sim$ provides a definition of the $N$-compact topology, it is important for a full understanding of this topology to obtain a description of the actual monads for this topology (see [14], for an elucidation of this point).

The first part of this paper is devoted to the investigation of the $N$-compact topology and a closely related topology we call the $S$ compact topology. With either of these topologies $\Gamma$ is compact and the one point compactification of $X$ is embedded as a closed subset of $\Gamma$ by the mapping $x \rightarrow\{x\}$ with $\infty$ corresponding to $\varnothing \in \Gamma$. When $X$ is locally compact, the $S$-compact and $N$-compact topologies are identical, both are Hausdorff, and the monad, $\mu(F)$, of a point $F \in \Gamma$ is given by $\mu(F)=\left\{H \in{ }^{*} \Gamma \mid F \sim H\right\}$. When $X$ is not locally compact the $S$ compact and $N$-compact topologies may be different, neither is Hausdorff and neither monad is given by $\left\{H \in{ }^{*} \Gamma \mid F \sim H\right\}$. The $S$-compact topology has a good standard as well as a good nonstandard characterization.

The technique Narens has developed suggests several different topologies on $\Gamma$. In the second section we use this technique to obtain a nice description of the Vietoris topology. This description elucidates some of the properties of the Vietoris topology. In the third section of the paper we define a new topology, called the fine topology on $\Gamma$. This topology has many nice properties and, in particular, may be regarded in some sense as the analog in the topological category of the Hausdorff metric (see Theorem III.8).

Throughout this paper, $X, Y$ and $Z$ will always denote Hausdorff spaces (although $\Gamma$ may not be Hausdorff). When we are dealing with several spaces $X, Y$ and $Z$, their extensions will always be taken in a single nonstandard model ${ }^{*} \mathcal{M}$. That is, we let $\mathcal{M}$ be the complete higher order structure on $X \cup Y \cup Z$ and let ${ }^{*} \mathcal{M}$ be a higher order elementary extension of $\mathcal{M}$ ([8], [9], [13]). If $\kappa$ is the cardinality of the universe of $\mathcal{M}$ we will assume throughout that ${ }^{*} \mathcal{M}$ is at least $\kappa^{+}$saturated ([1], [3], [4], [5], [11]). Thus we will assume $\mathrm{GCH}$ to insure that such an extension exists. 
I. The $N$-compact and $S$-compact topologies. Suppose throughout this section that $X$ is a Hausdorff space and that $\Gamma_{X}$ (or $\Gamma$ where confusion is unlikely) is the set of closed subsets of $X$. We topologize $\Gamma$ as follows

I.1. Definition. Suppose $F \in \Gamma$ and $H \in{ }^{*} \Gamma$ we write $H \sim F$ whenever $\operatorname{St}(H)=F$. Then the premonad, $m(F)$, of $F$ in ${ }^{*} \Gamma$ is defined by $m(F)=\left\{H \in{ }^{*} \Gamma \mid H \sim F\right\}$. A subset $A \subseteq \Gamma$ is said to be $N$-open if and only if for each $F \in A, m(F) \subseteq{ }^{*} A$. It is easy to verify that the $N$-open subsets of $\Gamma$ form a topology which, modifying the terminology of [12], we call the $N$-compact topology.

If $F \in \Gamma$ the monad, $\mu_{N}(F)$, of $F$ in the $N$-compact topology is defined in the usual way by

$$
\mu_{N}(F)=\bigcap_{F \in A, A N \text {-open }} * A
$$

It is immediate from these definitions that $m(F) \subseteq \mu_{N}(F)$ although it turns out (See I.9) that frequently $m(F) \neq \mu_{N}(F)$.

I.2. Lemma. Suppose $\mathcal{O}$ is an open subset of $X$. Define $\hat{O} \subseteq \Gamma$ by $\hat{O}=\{F \mid F \cap \hat{O} \neq \varnothing\}$. Then $\hat{O}$ is open in the N-compact topology.

Proof. Suppose $F \in \hat{O}$ and $H \sim F$. Since $F \in \hat{O}$ there is a point $x \in \mathcal{O} \cap F$. Since $\mathcal{O}$ is open $\mu(x) \subseteq{ }^{*} \mathcal{O}$. Since $\operatorname{St}(H)=F$, $x \in \operatorname{St}(H)$. Hence, $\mu(x) \cap H \neq \varnothing$ and thus ${ }^{*} O \cap H \neq \varnothing$. Therefore $H \in{ }^{*} \mathcal{O}$.

I.3. Lemma. Suppose $K$ is a compact subset of $X . \quad$ Define $\check{K} \subseteq \Gamma$ by $\check{K}=\{F \in \Gamma \mid F \cap K=\varnothing\}$. Then $K$ is open in the N-compact topology.

Proof. Suppose $F \in \check{K}$ and $H \sim F$. If $H \notin \check{K}$ then $H \cap{ }^{*} K \neq \varnothing$. Let $x \in H \cap{ }^{*} K$. Since $K$ is compact $y=\operatorname{St}(x)$ exists and is in $K$. Since $\operatorname{St}(H)=F, \quad y \in F$. Thus $K \cap F \neq \varnothing$ and $F \notin \check{K}$. This contradiction completes the proof. $T_{1}$.

I.4. Proposition. $\Gamma$ with the $N$-compact topology is compact and

Proof.

(i) If $H \in{ }^{*} \Gamma, H \in m(\mathrm{St}(H)) \subseteq \mu_{N}(\mathrm{St}(H))$. Thus every point of ${ }^{*} \Gamma$ is near-standard and $\Gamma$ is compact.

(ii) Suppose $F, G \in \Gamma, F \neq G$. Without loss of generality we may assume $F \backslash G \neq \varnothing$. Choose $a \in F \backslash G$. By Lemma I.3 $\{\breve{a}\}$ is open and 
$G \in\{\breve{a}\}$ but $F \notin\{\breve{a}\} . \quad$ By Lemma I.2 $\widehat{X \backslash G}$ is open and $G \notin \widehat{X \backslash G}$ while $F \in X \mid G$.

It is natural to ask when $\Gamma$ with the $N$-compact topology is Hausdorff. The following proposition shows this occurs precisely when the premonads, $m(F)$, are actually the monads, $\mu_{N}(F)$.

I.5. Proposition. The following are equivalent.

(i) $\Gamma$ is Hausdorff.

(ii) For each $F \in \Gamma, m(F)=\mu_{N}(F)$.

Proof. (ii) $\rightarrow$ (i) is immediate since $F \neq G$ implies $m(F) \cap m(G)=$ $\varnothing$.

(i) $\rightarrow$ (ii). We must show $\mu_{N}(F) \subseteq m(F)$. Suppose $H \notin m(F)$. Hence $\operatorname{St}(H) \neq F$. Since $\Gamma$ is Hausdorff $\mu_{N}(\operatorname{St}(H)) \cap \mu_{N}(F)=\varnothing$. But $H \in m(\mathrm{St}(H)) \subseteq \mu_{N}(\mathrm{St}(H))$ and, hence, $H \notin \mu_{N}(F)$. This completes the proof.

I.6. THEOREM. Suppose $X$ is locally compact then $\Gamma$ is Hausdorff and, hence, by I.5 for each $F \in \Gamma, m(F)=\mu_{N}(F)$.

Proof. Suppose $A, B \in \Gamma$. Without loss of generality we may assume $A \backslash B \neq \varnothing$. Choose $a \in A \backslash B$. Since $X$ is locally compact there is an open set $U$ such that $a \in U, \bar{U}$ is compact and $\bar{U} \cap B=\varnothing$. By Lemma I.2 $\hat{U}$ is open. BX Lemma I.3 $\check{U}$ is open. Clearly $\hat{U} \cap \check{U}=$ $\varnothing$. But $A \in \hat{U}$ and $B \in \widetilde{U}$ which completes the proof.

The converse of Theorem I.6 is also true as a consequence of the fact (Theorem I.8) that the one-point compactification of $X$ can be embedded in $\Gamma$ with the point at infinity corresponding to the empty set.

I.7. Definition. Let $X^{+}$denote the one-point compactification of $X$ with $\infty$ denoting the point at infinity. We define an embedding $e: X^{+} \rightarrow \Gamma$ by

$$
\begin{aligned}
& e(\infty)=\varnothing \\
& e(x)=\{x\} \quad \text { if } \quad x \neq \infty .
\end{aligned}
$$

I.8. THEOREM. $e: X^{+} \rightarrow \Gamma$ is a homeomorphism of $X^{+}$into $\Gamma$.

Proof. (i) Suppose $x \in X$. If $y \in \mu(x)$ then $\{y\} \sim\{x\}$ so ${ }^{*} e(y) \in m(e(x))$. Hence $e(\mu(x)) \subseteq m(e(x)) \subseteq \mu_{N}(e(x))$. Now, suppose $y \notin \mu(x)$ then there is a standard open set $\mathcal{O}$ such that $x \in \mathcal{O}$ and 
$y \notin * \mathscr{O}$. By Lemma $\mathrm{I} .2 \hat{\hat{O}}$ is open in $\Gamma$ and $e(x) \in \hat{O}$ but ${ }^{*} e(y) \notin \hat{\mathcal{O}}$. Hence, ${ }^{*} e(y) \notin \mu_{N}(e(x))$. Thus $e(\mu(x))=\mu_{N}(e(x))$.

(ii) We must show $\mu_{N}(\varnothing) \cap{ }^{*} e\left(X^{+}\right)=A$ where $A$ is given by $A=$ $\{\varnothing\} \cup\{\{x\} \mid x \in \mu(\infty)\}$. Notice that $\mu(\infty)=\bigcap_{K \text { compact }}{ }^{*}(X-K) \cup\{\infty\}$. By Lemma $I .3$ if $K$ is compact $\check{K}$ is open. But $\check{K} \cap e\left(X^{+}\right)=$ $\varnothing \cup e(X-K)$. Hence $\mu_{N}(\varnothing) \cap{ }^{*} e\left(X^{+}\right) \subseteq A$. Now suppose $\{y\} \notin \mu_{N}(\varnothing)$. Hence there is an open set $O \subseteq \Gamma$ s.t. $\varnothing \in \mathcal{O}$ but $\{y\} \notin^{*} \mathcal{O}$. Let $K=\{x \in X \mid\{x\} \notin \mathcal{O}\}$. Notice if $x \in{ }^{*} K$ then $\{x\} \notin^{*} \mathcal{O}$, so $\{x\} \notin m(\varnothing)$ and hence $x$ must be near-standard. Now, if $\{$ st $(x)\} \in \mathcal{O}$ then $\{x\}$ would also be in * $\mathcal{O}$ so $\{$ st $(x)\} \notin \mathcal{O}$ and $\operatorname{St}(x) \in K$. Thus, we've shown for each $x \in{ }^{*} K$, St $(x)$ exists and is in $K$, so $K$ is compact. Now $y \in{ }^{*} K$, so $y \notin \mu(\infty)$. Hence $A \subseteq \mu_{N}(\varnothing) \cap e\left(X^{+}\right)$which completes the proof.

I.9. COROllary. The following are equivalent

(i) $X$ is locally compact

(ii) $\Gamma$ is Hausdorff

(iii) For each $F \in \Gamma, m(F)=\mu_{N}(F)$.

Proof. Immediate from I.5, I.6 and I.8 since $X^{+}$is Hausdorff if and only if $X$ is locally compact.

We would like to obtain a standard description of the compact topology on $\Gamma$. Lemmas $\mathrm{I} .2$ and I.3 suggest a topology which is analogous to Vietoris topology. This approach is developed in the following pages. For locally compact spaces the two topologies are identical. However, for more general spaces they may be distinct (see Example I.16).

I.10. Definition. A subset $\mathcal{O}$ of $X$ is said to be cocompact whenever $X \backslash \mathcal{O}$ is compact. Suppose that $\mathcal{O}$ is cocompact and that $U_{1}, U_{2}, \cdots, U_{n}$ are open subsets of $X$. Then let $\left\langle\mathcal{O}, U_{1}, U_{2}, \cdots, U_{n}\right\rangle$ denote the set

$$
\left\{F \in \Gamma \mid F \subseteq \mathcal{O} \quad \text { and for } \quad i=1,2, \cdots, n \quad F \cap U_{i} \neq \varnothing\right\}
$$

By Lemmas I.2 and I.3 the set $\left\langle\mathcal{O}, U_{1}, U_{2}, \cdots, U_{n}\right\rangle$ is open in the $N$-compact topology. Let $\mathscr{B}$ denote the set of all such $\left\langle\mathcal{O}, U_{1}, U_{2}, \cdots, U_{n}\right\rangle$. Notice the intersection of two sets in $\mathscr{B}$ is again in $\mathscr{B}$

$$
\begin{aligned}
& \left\langle\mathcal{O}, U_{1}, U_{2}, \cdots, U_{n}\right\rangle \cap\left\langle\mathcal{O}^{\prime}, V_{1}, V_{2}, \cdots, V_{k}\right\rangle \\
& \quad=\left\langle\mathcal{O} \cap \mathcal{O}^{\prime}, U_{1}, U_{2}, \cdots, U_{n}, V_{1}, V_{2}, \cdots, V_{k}\right\rangle
\end{aligned}
$$


So, $\mathscr{B}$ forms a basis for a topology on $\Gamma$. This topology is called the $S$-compact topology. By the above remarks every set open in $S$-compact topology is also open in the $N$-compact topology. Hence, the $S$ compact topology is compact. Furthermore, an examination of the proof of Theorem I.8 shows that $e: X^{+} \rightarrow \Gamma$ is a homeomorphism of $X^{+}$ into $\Gamma$ with the $S$-compact topology.

One of the basic lemmas in Nonstandard Topology is that if $X$ is a topological space, $x \in X$ and $\mu(x)$ its monad then there is a *open set $U$ such that ${ }^{*} x \in U \subseteq \mu(x)$. For the $N$-compact topology we have been working primarily with the premonad $m(F)$ rather than the actual monad $\mu_{N}(F)$ of $F$ in the $N$-compact topology. It is not true that for this premonad there is always a ${ }^{*}$ open set $U$ in the $N$-compact topology such that ${ }^{*} F \in U \subseteq m(F)$. However, when. $X$ is locally compact $m(F)=$ $\mu_{N}(F)$ and such a $U$ can always be found. In particular the following lemma shows such a $U$ can be found in ${ }^{*} \mathscr{B}$.

I.11. Lemma. Suppose $X$ is locally compact and $F \in \Gamma$. Then there is a set $U \in{ }^{*} \mathscr{B}$ such that $F \in U \subseteq m(F)$.

Proof. (i) For each $x \notin F$ let $W_{x}$ be in an open set such that $x \in W_{x}, \bar{W}_{x}$ is compact and $\bar{W}_{x} \cap F=\varnothing$. By a straightforward enlargements argument there is *compact set $K$ such that $K \cap F=\varnothing$ and for each $x \notin F, \bar{W}_{k} \subseteq K$. Let $\mathscr{O}=X-K$.

(ii) For each $x \in F$ choose a ${ }^{*}$ open set $U_{x}$ such that $x \in U_{x} \subseteq$ $\mu(x)$. By a straightforward saturation argument there is an internal ${ }^{*}$ finite collection of ${ }^{*}$ open sets $\left\{V_{1}, V_{2}, \cdots, V_{\nu}\right\}$ such that for each $i=1,2, \cdots, \nu, V_{\imath} \cap{ }^{*} F \neq \varnothing$ and for each $x \in F, U_{x} \in\left\{V_{1}, V_{2}, \cdots, V_{\nu}\right\}$.

(iii) Let $U=\left\langle\mathcal{O}, V_{1}, V_{2}, \cdots, V_{\nu}\right\rangle \in * \mathscr{B}$. It is straightforward to verify that ${ }^{*} F \in U \subseteq m(F)$.

I.12. Corollary. Suppose $X$ is locally compact. Then $\mathscr{B}$ is a basis for the $N$-compact topology on $\Gamma$. Hence, the $S$-compact topology and $N$-compact topology are identical.

\section{Proof. Immediate from Lemma I.11.}

Example I.16 will show that the $S$-compact and $N$-compact topologies may be distinct when $X$ is not locally compact. In view of this fact if $F \in \Gamma$ we denote its monad in the $S$-compact topology by $\mu_{S}(F)$. Notice, $\mu_{N}(F) \subseteq \mu_{S}(F)$. In order to obtain a characterization of $\mu_{S}(F)$ we need a definition.

I.13. Definition. Suppose $X$ is a topological space and $x \in$ ${ }^{*} X . \quad x$ is said to be a far point provided for every standard compact 
subset $K$ of $X, x \notin^{*} K$. Let $\operatorname{FAR}(X)=\left\{x \in{ }^{*} X \mid x\right.$ is far $\}$, when $X$ is locally compact, the far points of ${ }^{*} X$ are precisely the nonnearstandard points.

I.14. Proposition. Suppose $F \in \Gamma$ and $H \in{ }^{*} \Gamma$ then $H \in \mu_{S}(F)$ if and only if

(i) $F \subseteq \mathrm{St}(H)$ and

(ii) For every $x \in H$ either $x \in \operatorname{FAR}(X)$ or $\operatorname{St}(x)$ exists and $\operatorname{St}(x) \in$ F.

Proof. ( $\rightarrow$ ) (i) Suppose $x \in F$ and $U$ is a standard open set with $x \in U$. Therefore $F \in\langle X, U\rangle$ so $H \in{ }^{*}\langle X, U\rangle$ and $H \cap$ ${ }^{*} U \neq \varnothing$. Hence, by a straighforward saturation argument $H \cap$ $\mu(x) \neq \varnothing$. So $x \in \operatorname{St}(H)$.

(ii) Suppose $x \in H$ and $x \notin \mathrm{FAR}(X)$. Therefore there is a standard compact set $K$ with $x \in{ }^{*} K$. Thus, St $(x)$ exists and $\operatorname{St}(x) \in K$.

Now suppose $\operatorname{St}(x) \notin F$. Since $K$ is compact there is an open subset $U$ of $K$ such that $\operatorname{St}(x) \in U$ and $\bar{U} \cap F=\varnothing . \quad \bar{U}$ is compact since it is a closed subset of $K$ and $x \in{ }^{*} \bar{U}$ since $\operatorname{St}(x) \in U$. Since $\bar{U} \cap F=$ $\varnothing, F \in\langle X \backslash \bar{U}\rangle$. But ${ }^{*} \bar{U} \cap H \neq \varnothing$, so $H \notin^{*}\langle X \backslash \bar{U}\rangle$ contradicting $H \in$ $\mu_{S}(F)$.

$(\leftarrow)$ Suppose $F \in\left\langle\mathcal{O}, U_{1}, \cdots, U_{n}\right\rangle$. First, suppose $H \not{ }^{*} \mathcal{O}$, then $H \cap{ }^{*}(X \backslash \mathcal{O}) \neq \varnothing$. So $t \in H \cap{ }^{*}(X \backslash \mathcal{O})$, St $(t) \in X \mid \mathcal{O}$; so $F \not{ }^{*} \mathcal{O}$, contradicting $F \in\left\langle\mathcal{O}, U_{1}, \cdots, U_{n}\right\rangle$. Now, $F \cap U_{1} \neq \varnothing$. Therefore there is an $x \in F \cap U_{1}$. But $\mu(x) \subseteq{ }^{*} U_{\text {l }}$ and by (i) there is a $y \in \mu(x) \cap H$. So $H \cap{ }^{*} U_{1} \neq \varnothing$. This completes the proof.

\section{I.15. Corollary.}

(a) Suppose $F \in \Gamma$ and $H \in \mu_{N}(F)$ then
(i) $F \subseteq \operatorname{St}(H)$ and
(ii) For every $x \in H$ either $x \in \operatorname{FAR}(X)$ or $\operatorname{St}(x)$ exists and $\operatorname{St}(x) \in F$.

(b) The mapping $u: \Gamma \times \Gamma \rightarrow \Gamma$ defined by $u(H, F)=H \cup F$ is continuous in the $S$-compact topology.

Proof.

(a) $\mu_{N}(F) \subseteq \mu_{S}(F)$.

(b) For $(H, F) \in \Gamma \times \Gamma, \mu(H, F)=\mu_{s}(H) \times \mu_{s}(F)$ and clearly $H^{\prime} \in \mu_{s}(H), F^{\prime} \in \mu_{s}(F)$ implies $H^{\prime} \cup F^{\prime} \in \mu_{s}(H \cup F)$.

By Corollary I.12 if $X$ is locally compact $u$ is continuous in the $N$-compact topology. However, without this assumption the author does not know whether $u$ is continuous in the $N$-compact topology. Clearly, if $H^{\prime} \in m(H)$ and $F^{\prime} \in m(F)$ then $H^{\prime} \cup F^{\prime} \in$ $m(H \cup F)$. However, although this provides some evidence for the continuity of $u$, it is not by itself sufficient to prove $u$ is continuous. 
The following example shows that the $N$-compact and $S$-compact topologies are distinct.

I.16. EXAMPLE. We first state carefully two facts necessary for this example.

(i) If $A \subseteq \Gamma$ then $A$ is closed in the $N$-compact topology if and only if for each $F \in{ }^{*} A, \operatorname{St}(F) \in A$. This equivalence is an immediate consequence of Definition I.1.

(ii) If $A \subseteq \Gamma$ then $A$ is closed in the $S$-compact topology if and only if for each $F \in{ }^{*} A$ and each $H \in \Gamma, F \in \mu_{S}(H)$ implies $H \in A$. Notice that since $\Gamma$ is not Hausdorff there may be many $H \in A$ such that $F \in \mu_{S}(H)$. This equivalence is an immediate consequence of Definition I.10.

Now let $Q$ denote the rationals and let $G$ denote the set of closed subgroups of $Q$. We claim $G$ is closed in the $N$-compact topology but not in the $S$-compact topology. The first assertion was proved by Narens in [12] by means of (i) above and the observation that if $F$ is a *closed *subgroup of a topological group then so is $\operatorname{St}(F)$. We proceed to the second assertion.

By a straightforward enlargement argument there is an $\alpha \in{ }^{*} Q$ such that

(i) $\alpha \in \mu(1)$

(ii) For each standard integer $n$

$$
n \alpha \in \operatorname{FAR}(Q) \text {. }
$$

Now let $H=\left\{n \alpha \mid n \in{ }^{*} Z\right\}$, where $Z$ denotes the set of integers. $H$ is clearly a closed subgroup of ${ }^{*} Q$. By Proposition I.14 $H \in \mu_{s}(\{1\})$. But $\{1\}$ is not a subgroup of $Q$. This completes the proof.

Although the $N$-compact and $S$-compact topologies on $\Gamma$ have some very nice properties they, also lack some desirable properties. In particular certain constructions on $\Gamma$ which one might like to be continuous are not continuous with these topologies. We close this section with several such examples before going on to discuss other topologies on $\Gamma$ in the remainder of this paper.

I.17. ExAmples. (i) Suppose $f: X \rightarrow Y$ is a continuous map. $f$ induces a map $\bar{f}: \Gamma_{X} \rightarrow \Gamma_{Y}$ defined by $\bar{f}(A)=\overline{f(A)}$. One might desire that $\bar{f}$ be continuous. However, this need not be so. In particular, if $\bar{f}$ were continuous this would imply that $f$ had a continuous extension to $g: X^{+} \rightarrow Y^{+}$with $g(\infty)=\infty$. No such extension exists, for example, if $f:(0,1) \rightarrow R$ is the usual inclusion of the open unit interval into the real line.

(ii) Suppose again that $f: X \rightarrow Y$ is continuous. $f$ induces a map 
$\hat{f}: \Gamma_{Y} \rightarrow \Gamma_{X}$ defined by $f(A)=f^{-1}(A)$. Again one might hope that $\hat{f}$ would be continuous. However, this need not be so. In particular if $f$ is a bijection then the continuity of $\hat{f}$ would imply $f$ is a homeomorphism which is, in general, false.

(iii) Let $X=R$ and define $f: \Gamma \rightarrow \Gamma$ by

$$
f(A)=\{x \in R \mid a \in A, a \leqq x\}
$$

$f$ is not continuous since if $\alpha$ is any negative infinite nonstandard real $\{\alpha\} \in \mu_{N}(\varnothing)$ but $f(\{\alpha\})=[\alpha, \infty) \notin \mu_{S}(\varnothing)=\mu_{S}(f(\varnothing))$.

II. The Vietoris topology. Some of the difficulties noted at the end of $\S I$ result from the fact that knowing $H \in \mu_{N}(F)$ gives us little or no information about the non-nearstandard points in $H$ and ${ }^{*} F$. In order to obtain a topology on $\Gamma$ which takes these points into consideration we need a notion of monads for points which are not nearstandard. One such notion is the coarse "monad system" defined in [14] and [15]. We suppose throughout this section that $X$ is a $T_{1}$ space.

II.1. Definition. Suppose $x \in{ }^{*} X$ the coarse monad of $x$, denoted $c(x)$ is defined by

$$
c(x)=\bigcap_{x \in \in^{*} F \complement^{*}, F_{U \text { standard closed, }}^{U \text { standard open }}} * U
$$

We collect some results about $c(x)$ in the following proposition.

II.2. Proposition.

(i) If $x \in X, c(x)=\mu(x)$

(ii) If $x, y \in{ }^{*} X, x \in c(y) \leftrightarrow y \in c(x)$

(iii) $X$ is regular $\leftrightarrow$ for every nearstandard $x, c(x)=\mu(\operatorname{St}(x))$. $\varnothing$.

(iv) $X$ is normal $\leftrightarrow$ if $x, y \in{ }^{*} X$ either $c(x)=c(y)$ or $c(x) \cap c(y)=$

(v) If $f: X \rightarrow Y$ is continuous then for every $x \in{ }^{*} X{ }^{*} f(c(x)) \subseteq$ $c\left({ }^{*} f(x)\right)$.

\section{Proof.}

(i) Clear.

(ii) If $x \notin c(y)$ then there is a standard closed set $F$ and a standard open set $U$ such that $y \in{ }^{*} F$ but $x \notin^{*} U$. But then $x \in{ }^{*}(X \backslash U)$ and $y \notin *(X \backslash F)$ so $y \notin c(x)$.

(iii) and (iv) see [14].

(v) Clear. 
II.3. Definition. Suppose $F \in \Gamma$ and $H \in{ }^{*} \Gamma$. We say $H \tau F$ whenever for every $x \in{ }^{*} F$ there is a $y \in H$ such that $y \in \bar{c}(x)$ and for every $y \in H$ there is an $x \in{ }^{*} F$ s.t. $y \in \bar{c}(x)$. The $c$-monad of $F, \bar{c}(F)$ is defined by $\bar{c}(F)=\left\{H \in{ }^{*} \Gamma \mid H \widetilde{c} F\right\}$. Notice, in particular, that $\bar{c}(\varnothing)=$ $\{\varnothing\}$.

II.4. Theorem. Suppose $F \in \Gamma$, then $\bar{c}(F)$ is the monad of $F$ in the Vietoris topology.

Proof. First recall that a basis for the Vietoris topology on $\Gamma$ is given by sets of the form $\left\langle\mathcal{O}, V_{1}, \cdots, V_{n}\right\rangle=\{F \in \Gamma \mid F \subseteq \mathcal{O}$ and for $i=$ $\left.1,2, \cdots, n F \cap V_{\imath} \neq \varnothing\right\}$ where $\mathcal{O}, V_{1}, \cdots, V_{n}$ are open subsets of $X$.

(i) Suppose $F \in\left\langle\mathcal{O}, V_{1}, \cdots, V_{n}\right\rangle$ and $H \in \bar{c}(F)$. For each $y \in H$ there is an $x \in{ }^{*} F$ such that $y \in c(x)$. Since $F$ is closed and $F \subseteq \mathcal{O}$ $c(x) \subseteq{ }^{*} \mathcal{O}$. Hence $H \subseteq{ }^{*} \mathcal{O}$. Since $F \in\left\langle\mathcal{O}, V_{1}, \cdots, V_{n}\right\rangle$ there is an $x \in$ $F \cap V_{i}$ for each $i$. Since $\{x\}$ is closed $c(x) \subseteq{ }^{*} V_{i}$ and since $H \approx F$ there is a $y \in H \cap c(x)$. Hence $H \cap{ }^{*} V_{1} \neq \varnothing$. Thus $H \subseteq{ }^{*}\left\langle\mathcal{O}, V_{1}, \cdots, V_{n}\right\rangle$.

(ii) Suppose $H \notin \bar{c}(F)$. There are two possibilities.

(a) For some $x \in{ }^{*} F$ there is no $y \in H$ such that $y \in$ $c(x)$. Hence, by a straightforward saturation argument there is a standard open $U$ and closed $T$ with $x \in{ }^{*} T \subseteq{ }^{*} U$ and ${ }^{*} U \cap H=$ $\varnothing$. But then $F \in\langle X, U\rangle$ and $H \notin *\langle X, U\rangle$.

(b) For some $y \in H$ there is no $x \in{ }^{*} F$ such that $x \in$ $c(y)$. Hence by a straightforward saturation argument there is a standard open $U$ and closed $T$ with $y \in{ }^{*} T \subseteq{ }^{*} U$ and $U \cap F=\varnothing$. But then $F \in\langle X \mid T\rangle$ and $H \notin^{*}\langle X \backslash T\rangle$.

Thus, in either case $H \notin \bar{c}(F)$ implies $H \notin$ monad of $F$ in the Vietoris topology, completing the proof.

Notice, that the mapping $X \rightarrow \Gamma$ defined by $x \rightarrow\{x\}$ is a homeomorphism into using the Vietoris topology but $e: X^{+} \rightarrow \Gamma$ is not even continuous (unless $X$ is compact) since $\varnothing$ is an isolated point of $\Gamma$ with the Vietoris topology. In addition it is clear that the mapping $u: \Gamma \times \Gamma \rightarrow \Gamma$ defined by $u(F, H)=F \cup H$ is continuous with the Vietoris topology.

With the Vietoris topology the mapping $\bar{f}$ defined in Example I.17 will be continuous if the range is normal. To see this we first observe that Definition II. 3 can be extended to the full * power set of $X$, denoted ${ }^{*} P(X)$.

II.5. Definition. Suppose $A, B \in{ }^{*} P(X)$. We say $A \approx B$ whenever for each $a \in A$ there is a $b \in B$ such that $a \in c(b)$ and for each $b \in B$ there is an $a \in A$ such that $a \in c(b)$. 
II.6. Lemma. Suppose $A \in{ }^{*} P(X)$ and $\bar{A}$ is the ${ }^{*}$ closure of $A$ then $A \approx \bar{A}$.

Proof. Since $A \subseteq \bar{A}$ we need only show that for each $x \in \bar{A}$ there is an $a \in A$ such that $a \in c(x)$. Suppose $x \in{ }^{*} F \subseteq{ }^{*} U$. $F$ standard closed and $U$ standard open. Since ${ }^{*} U$ is ${ }^{*}$ open and $x \in \bar{A}, A \cap$ ${ }^{*} U \neq \varnothing$. Hence, by a straightforward saturation argument $A \cap$ $c(x) \neq \varnothing$.

II.7. Proposition. Suppose $f: X \rightarrow Y$ is continuous, $Y$ is normal and $\bar{f}: \Gamma_{X} \rightarrow \Gamma_{Y}$ is defined by $\bar{f}(F)=\overline{f(F)}$. Then $\bar{f}$ is continuous in the Vietoris topology.

Proof. Since $Y$ is normal it is easy to see using Proposition II.2 (iv) that $\widetilde{c}$ is transitive on ${ }^{*} P(Y)$. From Proposition II.2 (v) it is clear that $F_{\tau} H$ implies $f(F) \tau f(H)$ but $\bar{f}(F) \approx f(F)$ and $\bar{f}(H) \tau f(H)$ by the preceding Lemma and, hence by transitivity $\bar{f}(F) \approx \bar{f}(H)$.

Although the coarse monad system imposes some control on the non-nearstandard points of $X$, this is not a very natural monad system, and, in fact, the coarse monads are much too large. As one result the Vietoris Topology has the following interesting property.

II.8. Monotone Limit Theorem. Suppose $F_{1} \subseteq F_{2} \subseteq \cdots$ is an ascending sequence in $\Gamma$. Let $F=\overline{\cup F}_{k}$. Then in the Vietoris topology $\operatorname{Lim}_{k \rightarrow \infty} F_{k}=F$.

Proof. Suppose $F \in\left\langle\mathcal{O}, U_{1}, \cdots, U_{n}\right\rangle$

(i) $F \subseteq \mathcal{O}$ implies $F_{k} \subseteq \mathcal{O}$ for each $k$

(ii) For each $i, F \cap U_{i} \neq \varnothing$. Hence there is an $x \in F \cap U_{l}$ and since $U_{t}$ is open $\left(\cup F_{k}\right) \cap U_{i} \neq \varnothing$. Therefore for some $k_{i}, F_{k_{i}} \cap$ $U_{i} \neq \varnothing$. Let $K=\max \left(k_{1}, \cdots, k_{n}\right)$.

Now for each $k \geqq K, F_{k} \in\left\langle\mathcal{O}, U_{1}, \cdots, U_{n}\right\rangle$.

From our point of view some insight into this theorem can be obtained from the following example.

II.9. ExAmple. Suppose $x \in{ }^{*} R$ is an infinite positive nonstandard real and $A$ is a standard set with $x \in{ }^{*} A$. Then $c(x) \cap{ }^{*} A$ contains arbitrarily small and large infinite numbers i.e. for each infinite positive $y \in{ }^{*} R, \quad c(x) \cap{ }^{*} A \cap(0, y) \neq \varnothing$ and $c(x) \cap{ }^{*} A \cap(-y, \infty) \neq \varnothing$. The proof of this is a straightforward saturation argument.

In the next section we consider a monad system on $X$ which gives more control over the non-nearstandard points. 
III. The fine topology. In [14], [15] we obtained a very natural monad system for non-nearstandard points. This monad system enables us to define a topology on $\Gamma$ which provides a great deal of control around non-nearstandard points. Throughout this section we will restrict our attention to normal spaces.

\section{III.1. Definition.}

(i) Suppose $U$ is a *open subset of ${ }^{*} X$ and $F$ is a ${ }^{*}$ closed subset of ${ }^{*} X$ with $F \subseteq U$. The pair $(U, F)$ is said to be a quasi-standard pair, Q.S.P. provided there is a standard locally finite collection $\mathcal{U}=$ $\left\{\left(U_{\alpha}, F_{\alpha}\right)\right\}_{\alpha \in \mathcal{I}}$ of pairs $\left(U_{\alpha}, F_{\alpha}\right)$ such that each $U_{\alpha}$ is open, each $F_{\alpha}$ is closed, $F_{\alpha} \subseteq U_{\alpha}$ and $(U, F) \in^{*} U$ (See [14], [15]).

(ii) If $x \in{ }^{*} X$ we define the monad of $x, \mu(x)$ by

$$
\mu(x)=\bigcap_{x \in F \subseteq U,(U, F) a Q . S P .} U .
$$

We recall the following facts about this monad system from [14], [15]. Our assumption that $X$ is normal is important here.

III.2. Propositions.

(i) If $x$ is standard, $\mu(x)$ is the usual monad $\mu(x)=$ $\bigcap_{x \in U, \text { Ustandardopen }} * U$

(ii) If $x$ is nearstandard $\mu(x)=\mu(\operatorname{St}(x))$

(iii) For each $x, y \in{ }^{*} X$ either $\mu(x)=\mu(y)$ or $\mu(x) \cap \mu(y)=\varnothing$.

(iv) If $f: X \rightarrow Y$ is continuous then for every $x \in{ }^{*} X,{ }^{*} f(\mu(x)) \subseteq$ $\mu\left({ }^{*} f(x)\right)$.

\section{Proof. [14], [15].}

III.3. Definition. Suppose $H, F \in{ }^{*} \Gamma$ we say $H_{\widetilde{\mu}} F$ whenever for every $x \in H, \mu(x) \cap F \neq \varnothing$ and for every $y \in F, \mu(y) \cap H \neq \varnothing$. In view of Proposition III. $2 \widetilde{\mu}$ is an equivalence relation. For each $F \in{ }^{*} \Gamma$, $\bar{\mu}(F)$ is given by $\bar{\mu}(F)=\left\{H \in{ }^{*} \Gamma \mid H_{\widetilde{\mu}} F\right\}$. The topology defined by the $\bar{\mu}$ monads is called the fine topology on $\Gamma$.

We can obtain a standard characterization of the fine topology in the obvious way.

III.4. Definition. Suppose $\mathcal{U}=\left\{U_{\alpha}\right\}_{\alpha \in \mathscr{I}}$ is a locally finite collection of open subsets of $X$ and $\mathcal{O}$ is an open subset of $X$. Then define $\langle\mathcal{O}, \mathcal{U}\rangle=\left\{F \in \Gamma \mid F \subseteq \mathcal{O}\right.$ and for each $\left.U_{\alpha} \in \mathcal{U}, U_{\alpha} \cap F \neq \varnothing\right\}$. Let $\mathscr{F}=$ $\left\{\langle\mathcal{O}, \mathcal{U}\rangle \mid \mathcal{O} \subseteq X\right.$ open, $\mathcal{U}=\left\{U_{\alpha}\right\}_{\alpha \in \mathscr{I}}$ a locally finite family of open sets in $X\}$.

III.5. THEOREM. $\mathscr{F}$ is a basis for the fine topology on $\Gamma$. 


\section{Proof.}

(i) Suppose $F \in \Gamma, F \in\langle\mathcal{O}, \mathcal{U}\rangle$ and $H \in \bar{\nu}(F)$. We must show that $H \in{ }^{*}\langle\mathcal{O}, \mathcal{U}\rangle$.

(a) $F \in\langle\mathcal{O}, \mathcal{U}\rangle$ implies $F \subseteq \mathcal{O}$. Hence for each $x \in F, \mu(x) \subseteq$ ${ }^{*} \mathcal{O}$. So, clearly $H \subseteq{ }^{*} \mathcal{O}$.

(b) For each $U_{\alpha} \in \mathcal{U}$ choose $x_{\alpha} \in U_{\alpha} \cap F$. Given $U_{\beta} \in{ }^{*} \mathcal{U}$ $\left(U_{\beta},\left\{x_{\beta}\right\}\right)$ is a Q.S.P. so $\mu\left(x_{\beta}\right) \subseteq U_{\beta}$ and since $\mu\left(x_{\beta}\right) \cap H \neq \varnothing$, $U_{\beta} \cap H \neq \varnothing$.

(ii) Suppose $F \in \Gamma$, and $H \notin \bar{\mu}(F)$. We must find an $\mathcal{O}, \mathcal{U}$ such that $F \in\langle\mathcal{O}, \mathcal{U}\rangle$ but $H \notin *\langle\mathcal{O}, \mathcal{U}\rangle$.

(a) Suppose for some $x \in{ }^{*} F, \mu(x) \cap H=\varnothing$. Then by a straightforward saturation argument there is a locally finite collection of pairs $U=\left\{\left(U_{\alpha}, F_{\alpha}\right)\right\}_{\alpha \in \mathscr{P}}$ containing a pair $\left(U_{\beta}, F_{\beta}\right) \in * \mathscr{U}$ such that $x \in F_{\beta}$ and $U_{\beta} \cap H=\varnothing$. Let $\mathscr{V}=\left\{U_{\alpha} \mid F \cap U_{\alpha} \neq \varnothing\right\}$. Then $F \in\langle X, \mathscr{V}\rangle$ but $H \notin\langle X, \mathscr{V}\rangle$ since $U_{\beta} \in{ }^{*} \mathscr{V}$.

(b) Suppose for some $y \in H, \mu(y) \cap F=\varnothing$. By a straightforward enlargement argument there is a locally finite collection of pairs $U=$ $\left\{\left(U_{\alpha}, F_{\alpha}\right)\right\}_{\alpha \in \Phi}$ such that for some $\beta, y \in F_{\beta}$ but $U_{\beta} \cap{ }^{*} F=\varnothing$. Let $T=\cup_{\alpha \in \Phi, F_{\alpha} \cap F=\varnothing} F_{\alpha}$. Since $U$ is locally finite, $T$ is closed. But $F \in$ $\langle X \backslash T, \varnothing\rangle$ and $H \notin *\langle X \backslash T, \varnothing\rangle$.

This completes the proof.

III.6. Proposition. Suppose $f: X \rightarrow Y$ is continuous and $\bar{f}: \Gamma_{X} \rightarrow \Gamma_{Y}$ is defined by $\bar{f}(F)=\overline{f(F)}$. Then $\bar{f}$ is continuous in the fine topology.

Proof. Entirely analogous to that of Proposition II.7.

Notice that as with the Vietoris topology the mapping $x \rightarrow\{x\}$ is a homeomorphism into of $X$ into $\Gamma$ but $e: X^{+} \rightarrow \Gamma$ is not (unless $X$ is compact) since $\varnothing$ is an isolated point of $\Gamma$. In addition the mapping $u: \Gamma \times \Gamma \rightarrow \Gamma$ given by $u(F, H)=F \cup H$ is easily seen to be continuous in the fine topology. It is easy to see that the Monotone Limit Theorem is false in the fine topology. In fact a counterexample is provided by the sequence $F_{n}=[-n, n]$ of subsets of $\mathbf{R}$.

In general, the compact topology is coarser than the Vietoris topology which in turn is coarser than the fine topology. Of course, when $X$ is compact all three topologies are identical.

For metric spaces the Hausdorff metric provides a very natural topology on $\Gamma$. One difficulty with this topology, however, is that it dependes in an essential way on the metric on $X$. Recall the definition of the Hausdorff metric.

III.7. Definition. Suppose $X$ is a metric space with metric $d$. 
(i) If $x \in X$ and $F \in \Gamma$ define

$$
\begin{aligned}
& \rho^{\prime}(x, F)=\inf _{y \in F} d(x, y) \text { if } F \neq \varnothing \\
& =\infty \quad \text { if } \quad F=\varnothing
\end{aligned}
$$

(ii) If $A, B \in \Gamma$ define

$$
\rho^{\prime}(A, B)=\max \left(\sup _{a \in A} \rho^{\prime}(a, B), \sup _{b \in B} \rho^{\prime}(b, A)\right) .
$$

Finally we define the Hausdorff metric $\rho_{d}(A, B)$ by $\rho_{d}(A, B)=$ $\min \left(1, \rho^{\prime}(A, B)\right)$. (See, for example [2] or [6] and [7]).

Since this topology depends in an essential way on the given metric $d$, it is natural to look for a topology related to the Hausdorff metric which is independent of the metric $d$.

III.8. Definition. Suppose $X$ is a metrizable space. If $d$ is a metric on $X$ let $\tau_{d}$ denote the set of open subsets of $\Gamma$ with the metric $\rho_{d}$. Let $\mathscr{B}=\bigcup_{d \text { ametricon } X} \tau_{d} . \quad \mathscr{B}$ is clearly a basis for a topology on $\Gamma$.

III.8. THEOREM. Suppose $X$ is metrizable space. Then $\mathscr{B}$ is a basis for the fine topology on $\Gamma$.

\section{Proof.}

(i) Suppose $F \in A \in \tau_{d}$. We must show $\bar{\mu}(F) \subseteq^{*} A$. Since $A \in \tau_{d}$ there is a standard $\epsilon>0$ such that for every $H \in{ }^{*} \Gamma,{ }^{*} \rho_{d}\left(H,{ }^{*} F\right)<$ $\epsilon$ implies $H \in{ }^{*} A$. But, now by [14, Theorem 2.12] for every $x, y \in{ }^{*} X$, $y \in \mu(x)$ implies ${ }^{*} d(x, y) \sim 0$. Hence, $H \in \bar{\mu}(F)$ implies ${ }^{*} \rho_{d}\left(H,{ }^{*} F\right) \sim$ 0 ; so $H \in{ }^{*} A$. Thus $\bar{\mu}(F) \subseteq \cap_{A \in \mathscr{B}}{ }^{*} A$.

(ii) Suppose $H \notin \bar{\mu}(F)$ we must find some $A \in \mathscr{B}$ such that $F \in A$ but $H \notin^{*} A$. Since $H \notin \bar{\mu}(F)$ there is an open set $\mathcal{O}$ and a locally finite collection $\mathscr{U}=\left\{U_{\alpha}\right\}_{\alpha \in \mathcal{F}}$ of open sets such that $F \in\langle\mathcal{O}, \mathcal{U}\rangle$ but $H \notin *\langle\mathcal{O}, \mathcal{U}\rangle$. There are two cases.

(a) $H \notin{ }^{*} \mathcal{O}$. Since $F$ is closed and $F \subseteq \mathcal{O}$ there is a continuous function $\sigma: X \rightarrow[0,1]$ such that $\sigma(x)=0$ for $x \in F$ and $\sigma(x)=1$ for $x \notin \mathcal{O}$. Let $d$ be any metric on $X$. Define a new metric $\delta$ on $X$ by $\delta(x, y)=d(x, y)+|\sigma(x)-\sigma(y)|$. It is straightforward to verify that $\delta$ is a metric on $X$. But if $z \in H \backslash^{*} \mathcal{O}$ and $x \in{ }^{*} F, \delta(x, y) \geqq 1$; so $\rho_{\delta}^{*}\left(H,{ }^{*} F\right) \geqq 1$ and, thus, $H \notin^{*}\left\{T \mid \rho_{\delta}(T, F)<1\right\}$ which is in $\tau_{\delta}$ and, hence, $\mathscr{B}$.

(b) For some $\beta \in E^{*} \mathscr{I}, \quad H \cap U_{\beta}=\varnothing$. For each $\alpha$ choose $x_{\alpha} \in U_{\alpha} \cap F$ and choose a continuous function $\sigma_{\alpha}: X \rightarrow[0,1]$ so that $\sigma_{\alpha}\left(x_{\alpha}\right)=1$ and $\sigma_{\alpha}(y)=0$ if $y \notin U_{\alpha}$. Let $d$ be any metric on $X$ and define a new metric $\delta$ on $X$ by $\delta(x, y)=d(x, y)+\max _{\alpha}\left|\sigma_{\alpha}(x)-\sigma_{\alpha}(y)\right|$. 
Notice for $y \in H,{ }^{*} \delta\left(x_{\alpha}, y\right) \geqq 1$ so ${ }^{*} \rho_{\delta}\left({ }^{*} F, H\right) \geqq 1$. Therefore, we need only verify that $\delta$ is a metric on $X$. But this is a straightforward verification after noticing that for given $x$ and $y$ there are neighborhoods $U$ of $x$ and $V$ of $y$ in which only finitely many $\sigma_{\alpha}$ 's are nonzero and, thus, $\max _{\alpha}\left|\sigma_{\alpha}(x)-\sigma_{\alpha}(y)\right|$ is continuous.

In view of Theorem III.8 the fine topology may be regarded in some sense as the analog of the Hausdorff metric in the topological category.

\section{REFERENCES}

1. J. L. Bell and A. B. Slomson, Models and Ultraproducts: an Introduction, North-Holland, Amsterdam, 1969.

2. F. Hausdorff, Mengenlehre, Dover, New York. (Translation) Set Theory, Chelsea, New York, 1957.

3. H. J. Keisler, Good ideals in fields of sets, Ann. of Math., (2) 79 (1964), 338-359.

4. - Ultraproducts and elementary classes, Nederl. Akad. Wetensch. Proc. Ser. A $64=$ Indag. Math., 23 (1961), 477-495.

5. - Ultraproducts and saturated models, Nederl. Akad. Wetensch. Proc. Ser. A $67=$ Indag. Math., 26 (1964), 178-186.

6. K. Kuratowski, Topology, Vol. I, Academic Press, New York, 1966.

7. - Topology, Vol. II, Academic Press, New York, 1968.

8. W. A. J. Luxemberg, A new approach to the theory of monads, California Institute of Technology, Pasadena, Calif., 1967.

9. M. Machover and J. Hirschfeld, Lectures on nonstandard analysis, Lecture Notes in Math., Vol. 94, Springer-Verlag, New York, 1969.

10. E. Michael, Topologies on spaces of subsets, Trans. Amer. Math. Soc., 71 (1951), 152-182.

11. M. Morley and R. Vaught, Homogeneous universal models, Math. Scand., 11 (1962), 37-57.

12. L. Narens, Topologies of closed subsets, Trans. Amer. Math. Soc., 174 (1972), 55-76.

13. A. Robinson, Non-standard Analysis, North-Holland, Amsterdam (1974).

14. F. Wattenberg, Nonstandard topology and extensions of monad systems to infinite points, J. Symbolic Logic, 36 (1971), 463-476.

15. - Monads of infinite points and finite product spaces, Trans. Amer. Math. Soc., 176 (1973), $351-368$.

Received May 25, 1976.

UNIVERSITY OF MASSACHUSETTS

Amherst, MA 01002 



\section{PACIFIC JOURNAL OF MATHEMATICS}

\section{EDITORS}

RichaRd ARENS (Managing Editor)

University of California

Los Angeles, CA 90024

\section{R. A. Beaumont}

University of Washington

Seattle, WA 98105

C. C. Moore

University of California

Berkeley, CA 94720
J. DugunduI

Department of Mathematics

University of Southern California

Los Angeles, CA 90007

R. Finn AND J. Milgram

Stanford University

Stanford, CA 94305

\section{ASSOCIATE EDITORS}
E. F. BECKENBACH
B. H. NeumanN
F. WOLF
K. YoshidA

\section{SUPPORTING INSTITUTIONS}

UNIVERSITY OF BRITISH COLUMBIA

CALIFORNIA INSTITUTE OF TECHNOLOGY

UNIVERSITY OF CALIFORNIA

MONTANA STATE UNIVERSITY

UNIVERSITY OF NEVADA

NEW MEXICO STATE UNIVERSITY

OREGON STATE UNIVERSITY

UNIVERSITY OF OREGON

OSAKA UNIVERSITY

\author{
UNIVERSITY OF SOUTHERN CALIFORNIA \\ STANFORD UNIVERSITY \\ UNIVERSITY OF HAWAII \\ UNIVERSITY OF TOKYO \\ UNIVERSITY OF UTAH \\ WASHINGTON STATE UNIVERSITY \\ UNIVERSITY OF WASHINGTON \\ AMERICAN MATHEMATICAL SOCIETY
}

The Supporting Institutions listed above contribute to the cost of publication of this Journal, but they are not owners or publishers and have no responsibility for its contents or policies.

Mathematical papers intended for publication in the Pacific Journal of Mathematics should be in typed form or offset-reproduced (not dittoed), double spaced with large margins. Underline Greek letters in red, German in green, and script in blue. The first paragraph or two must be capable of being used separately as a synopsis of the entire paper. Items of the bibliography should not be cited there unless absolutely necessary, in which case they must be identified by author and Journal, rather than by item number. Manuscripts, in duplicate, may be sent to any one of the four editors. Please classify according to the scheme of Math. Reviews, Index to Vol. 39. All other communications should be addressed to the managing editor, or Elaine Barth, University of California, Los Angeles, California, 90024.

100 reprints are provided free for each article, only if page charges have been substantially paid. Additional copies may be obtained at cost in multiples of 50 .

The Pacific Journal of Mathematics is issued monthly as of January 1966. Regular subscription rate: $\$ 72.00$ a year (6 Vols., 12 issues). Special rate: $\$ 36.00$ a year to individual members of supporting institutions.

Subscriptions, orders for back numbers, and changes of address should be sent to Pacific Journal of Mathematics, 103 Highland Boulevard, Berkeley, California, 94708.

PUBLISHED BY PACIFIC JOURNAL OF MATHEMATICS, A NON-PROFIT CORPORATION

Printed at Jerusalem Academic Press, POB 2390, Jerusalem, Israel. 


\section{Pacific Journal of Mathematics}

Vol. 68, No. 2

April, 1977

William Allen Adkins, Aldo Andreotti and John Vincent Leahy, An analogue of Oka's theorem for weakly normal complex spaces ........

Ann K. Boyle, M. G. Deshpande and Edmund H. Feller, On nonsingularly

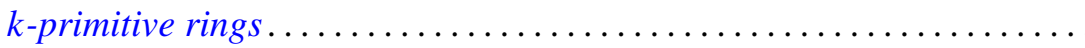

Rolando Basim Chuaqui, Measures invariant under a group of

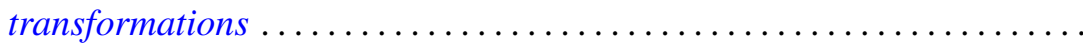

Wendell Dan Curtis and Forrest Miller, Gauge groups and classification of bundles with simple structural group .......................

Garret J. Etgen and Willie Taylor, The essential uniqueness of bounded nonoscillatory solutions of certain even order differential equations

Paul Ezust, On a representation theory for ideal systems

Richard Carl Gilbert, The deficiency index of a third order operator ........

John Norman Ginsburg, $S$-spaces in countably compact spaces using Ostaszewski's method.

Basil Gordon and S. P. Mohanty, On a theorem of Delaunay and some

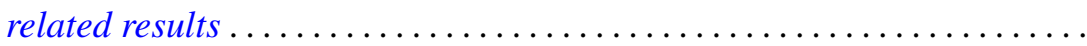

Douglas Lloyd Grant, Topological groups which satisfy an open mapping

theorem.

Charles Lemuel Hagopian, A characterization of solenoids

Kyong Taik Hahn, On completeness of the Bergman metric and its

subordinate metrics. II .

G. Hochschild and David Wheeler Wigner, Abstractly split group extensions.

Gary S. Itzkowitz, Inner invariant subspaces ...............

Jiang Luh and Mohan S. Putcha, A commutativity theorem for

non-associative algebras over a principal ideal domain.

Donald J. Newman and A. R. Reddy, Addendum to: "Rational approximation of $e^{-x}$ on the positive real axis".....

Akio Osada, On the distribution of a-points of a strongly annular function ....

Jeffrey Lynn Spielman, A characterization of the Gaussian distribution in a Hilbert space. .

Robert Moffatt Stephenson Jr., Symmetrizable-closed spaces ...

Peter George Trotter and Takayuki Tamura, Completely semisimple inverse $\Delta$-semigroups admitting principal series . . . . . . . .

Charles Irvin Vinsonhaler and William Jennings Wickless, Torsion free abelian groups quasi-projective over their endomorphism rings...

Frank Arvey Wattenberg, Topologies on the set of closed subsets ... 